\title{
DIVISION XI: SPACE AND HIGH ENERGY ASTROPHYSICS
}

\section{(ASTROPHYSIQUE SPATIALE ET DES HAUTES ENERGIES)}

\author{
PRESIDENT: G. Srinivasan \\ VICE-PRESIDENT: H. Okuda \\ BOARD: M.D. Arnaud, S.L. Baliunas, S.A. Bludman, T.J.L. Courvoisier, \\ G.S. Da Costa, H. Hasan, G. Hasinger, P.T. O'Brien, L. Piro, R.T. Schilizzi, \\ B.M. Shustov, W. Wamsteker \& Z. Wang
}

Commission 44: Space and High Energy Astrophysics

\section{General Activities}

Division XI has only one Commission associated with it,viz., Commission 44 on Space and High Energy Astrophysics. But the name derives from the fact that at the IAU General Assembly in the Hague (1994) it was decided to merge Commission 44 and Commission 48 (which dealt with High Energy Astrophysics). So the merged Commission covers a very broad spectrum of activities. And that makes the preparation of a tri-annual report for the Transactions a daunting task. Besides, given the pace at which the exploration of the Universe from space is proceeding one wonders at the efficacy of such reports. Most of the members of Board of Division XI were in favour of a short report. And so that is what it is going to be.

The Golden Age of astronomy from space continues, and the Table listing the various missions planned bears testimony to this. The "vertical line" indicates the time of preparation of this report (October, 2002). It is truly impressive that astronomy from space now not only embraces all the traditional areas from Radio Astronomy to Gamma Ray Astronomy but includes missions related to particle astrophysics and gravitational wave detectors!

\subsection{Working Group on Particle Astrophysics}

During the last few decades we have witnessed unprecedented progress in astronomy and astrophysics; with the advent of the space age several new windows to the Universe were opened. If one were to characterize these past decades, one would say that this has been the era of high energy astrophysics: of supernovae, neutron stars and black holes. At the dawn of the new millennium there is considerable excitement about several new initiatives that will herald in a new era. An era of astrophysics with ultra high energy gamma rays and cosmic rays; neutrino astronomy; the detection of gravitational waves; and the search for the ubiquitous dark matter.

While this excitement is prevalent in the astronomical community, it is not adequately reflected in the activities of the International Astronomical Union, such as its Joint Discussions, Colloquia and Symposia. The Executive of the IAU has requested Division XI to remedy this situation. With this objective in mind Division XI has set up a Working Group consisting of leading people working broadly in the areas mentioned above, viz. Ultra High Energy Gamma Rays and Cosmic Rays, Neutrino Astrophysics, Gravitational Wave detection and the search for Dark Matter. This Working Group is Chaired by Marco Salvati of the Arcetri Observatory and the full membership can be found at the IAU Website. 


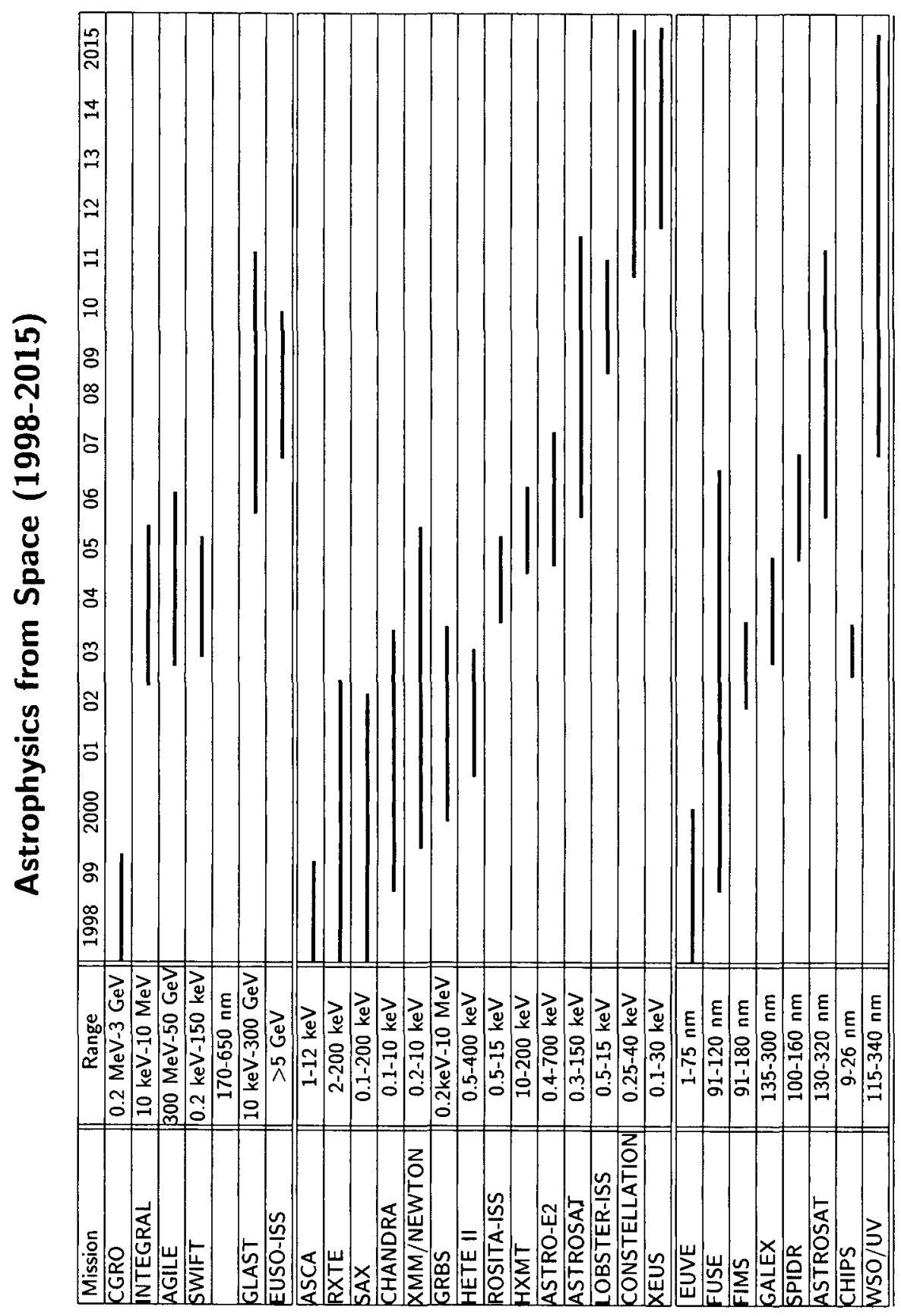




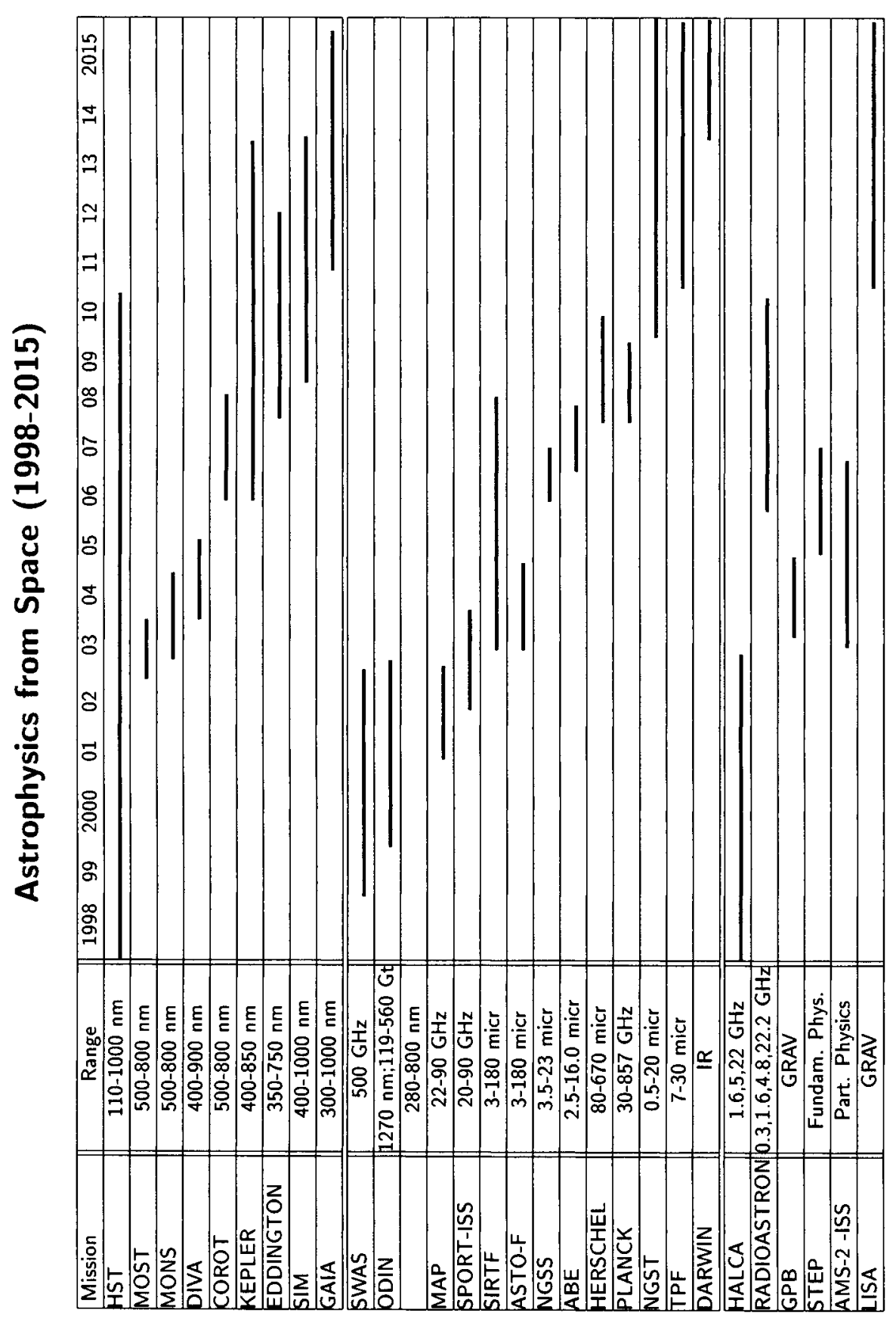


It may be worth recalling why these frontier areas are under-represented in the IAU fora. Historically speaking, investigators in these areas tended to have much closer ties with the physics community than the astronomical community. Indeed, by and large they are associated with IUPAP or COSPAR, rather than with the IAU (this is another thing that the proposed Working Group and Division XI can rectify!). It is our hope that this Working Group will play a vital role in better integrating these frontier areas into the folds of astronomy and astrophysics. As a beginning, this new Working Group will be organizing a Joint Discussion during the forthcoming General Assembly in Sydney in 2003.

Having said this, I should hasten to add that it is not the intention to 'pry' these activities out of the IUPAP or COSPAR. Any apprehension in this regard can be laid to rest by taking the case of Radio Astronomy. When radio astronomy came of age (soon after the second World War) its practitioners were mostly associated with the International Union of Radio Sciences (URSI). Today the Division on Radio Astronomy is one of the largest Divisions of the IAU. The respective divisions of the URSI and the IAU complement and supplement each other. A similar situation arose with the advent of high energy astronomy, viz., X-ray and Gamma ray astronomy. Initially, the major results were presented mainly at COSPAR meetings. Today, all these activities are integrated into Commission 44 and Division XI. We are sure that one can look forward to the same kind of synergy in the areas mentioned above between the IAU on the one hand and the IUPAP and COSPAR on the other.

\subsection{Working Group on Astronomy from the Moon}

This large Working Group consisting of nearly 30 members, and Chaired by N. Kaifu, was organized to explore the possibility of various astronomical observations from the Lunar surface. This is motivated by the realization that the Moon is a good site to do astronomy from (!), although one is talking of a fairly long time scale to realize this.

This Working Group has been quite active, and will be holding a scientific session during the General Assembly in Sydney next year. This meeting will cover a comprehensive list of topics, including Reviews of Science on the Moon, Missions to the Moon ( SMART1, LUNAR-A, SELENE, RISE etc.), Future plans (including Lunar science, astrometry, Optical/IR Monitors, Radio astronomy, SETI etc.).

\section{VLBI Space Observatory Program (VSOP)}

A status report, August 2002: The VSOP mission is operating since 12 Feb 1997. The HALCA satellite launched by the Institute of Space and Astronautical Science (ISAS, Japan) is the first dedicated VLBI radio telescope in Space. It is equipped with an 8-m diameter parabolic antenna, radio astronomy receivers operating at $1.6,5$ and $22 \mathrm{GHz}$, and data handling and down-link electronics. More than 25 ground-based radio telescopes in all continents are involved in VSOP observations. The projected life-time of the mission was three years. Until February 2002 the mission operated mainly as an astronomical facility available to the world-wide scientific community on the basis of peer-reviewed proposals. More than 500 observations were conducted as part of the VSOP open access time. Since February 2002, the mission operates as a tool for VSOP Survey of sub-milliarcsecond structures in extragalactic radio sources led by the leading institute of the mission, ISAS. In the framework of the VSOP Survey, about 300 extragalactic sources will be imaged with angular resolution unachievable from Earth. In addition to the network of ground-based radio telescopes, the mission includes as operational elements 5 special wide-band tracking stations (one remains operational after February 2002), three VLBI correlators (two remain operational after February 2002), a navigation support system. More information on the status and results of the VSOP mission is available at http://www.vsop.isas.ac.jp. 


\section{The Solar Neutrino Puzzle}

The year 2001 was a momentous year for the astroparticle physics community. The Solar Neutrino puzzle (the observed deficit), which perplexed both the physics and the astronomical community for three decades, may have been solved. This problem was particularly intriguing with regard to the Boron 8 neutrino flux, which is extremely sensitive to the conditions that obtain in the central regions of the Sun.

First, the astrophysics community fine tuned the solar model by including more precisely certain physical processes such as the contribution of the heavy elements to the opacities, latest reaction rates, screening effects, microscopic diffusion etc.. Secondly, helioseismology was invoked to check the correctness of the models of stellar structure. With the instruments GOLF and MDI onboard the satellite SOHO, they were able to study low amplitude acoustic modes with impressive precision. Using these modes, which are less perturbed by the turbulence in the outer regions, as well as solar cycle variations (Bertello et al. 2000; Garcià et al. 2001), astronomers were able to extract the sound speed profile in the inner core down to 0.07 solar radius, where these neutrinos are emitted (Basu et al. 2000; Turck-Chièze et al. 2001a). By doing so they have been able to reject phenomena which may lower the predicted flux of Boron 8 neutrinos, such as the effect on the reaction rates due to mixing and large screening effects. This led to a new model compatible with the profile of the sound speed which, in turn, was used to predict the neutrino fluxes (Turck-Chièze et al. 2001a, 2001b). The influence of the magnetic field in the central region was also excluded (Couvidat et al. 2002).

In parallel, the particle physics community concentrated their effort in extracting the Boron 8 neutrino flux by two techniques: the interaction of the neutrinos with water and heavy water, respectively. In the first method applicable to Superkamiokande (Couvidat et al. 2002), it is not possible to differentiate the electron neutrinos from the other species. Indeed, this led to the confirmation of the "deficit". But in the second method, the SNO detector, three interactions have been measured: elastic scattering, charge current and neutral current. Consequently, it has been possible to distinguish the different species, and apply to them the appropriate cross section. By doing this, and using the statistical significance of Superkamiokande, it has been possible to sum the contribution coming from electron neutrinos and the other species (Fukuda et al. 2001; Ahmad et al., 2001a, 2001b, 2001c).

This sum, $[5.09 \pm 0.4$ (stat) \pm 0.4 (systematic) $] \times 10^{6} \mathrm{~cm}^{2} \mathrm{~s}^{-1}$, is in excellent agreement with that predicted by Helioseismology, viz., $(4.98 \pm 0.7) \times 10^{6} \mathrm{~cm}^{2} \mathrm{~s}^{-1}$

The resolution of this major problem was possible largely due to the synergy that has existed between the physics and the astronomy communities. The agreement mentioned above may be considered as a "proof" of neutrino oscillations. The next step is to discover the properties of the neutrinos. Here, again, progress will depend upon the close interaction between the two communities.

\section{References}

Bertello, L, Varadi F., Ulrich, R. K., Henney, C. J., Kosovishev,, A. G., Garcia, R., TurckChièze, S, 2000, ApJ. Lett., 537 L 143

Garcià, R. A., Règulo, C., Turck-Chièze, S., Bertello, L., Kosovichev, A.G., Brun, A.S., Couvidat, S., Henney, C. J.Lazrek, M., Ulrich, R. K., Varadi, F., 2001, Sol. Phys., $200,361$.

Basu, S., Turck-Chièze S., Berthomieu, G., et al,ApJ, 2000, 535, 1078.

Turck-Chièze, S., Couvidat, S., Kosovichev, A. G., Gabriel, A. H., Berthomieu, G., Brun, A. S., Christensen-Dalsgaard, J., Garci, R. A., Gough, D. O., Provost, J., Roca-Corts, T., Roxburgh, I. W., and Ulrich, R. K., 2001a, ApJ 555, L69.

Turck-Chièze, S., Nghiem, P., Couvidat, S., and Turcotte, S., 2001b, Sol. Phys.(2001) 200, 323. 
Couvidat, S., Turck-Chièze, S., Kosovichev, S., (2002), ApJ preprint, astro-ph/0203107

Fukuda, Y., et al., 2001, Phys. Rev. Lett., 86, 5651

Ahmad, Q. R. et al; SNO collaboration, 2001a, Phys. Rev. Lett., 87, 071301

Ahmad, Q. R. et al;, SNO collaboration, 2001b, preprint, nucl-ex/0204008

Ahmad, Q. R. et al; SNO collaboration, 2001c, preprint, nucl-ex/0204009

\section{Ultra High Energy Cosmic Rays}

The detection of cosmic rays with energy in excess of $10^{20} \mathrm{eV}$ has remained an embarrassing puzzle for forty years now. The results from the largest experiment so far, AGASA, in Japan, has provided strong evidence for the existence of these events. No evidence was found for the so-called Greisen-Zatsepin-Kuzmin (GZK) cutoff. Nevertheless, any strong conclusion had to contend with the very low statistics of events at these extremely high energies. This challenge has led to the building of bigger experimental facilities. The HiRes collaboration has recently published the results of the last several years of investigation. The operation of the HiRes experiment has unfortunately been stopped after the tragic events of September 11,2001 since the experiment is located in a military zone. The unpleasant surprise that the present results provide is that they seem to be in agreement with the existence of the GZK cutoff in the cosmic ray spectrum, although the total number of events detected above $10^{19} \mathrm{eV}$ is lower than that of AGASA (Abu-Zayyad et al. 2002). Much effort is being channeled into the attempt to understand the origin of the disagreement between HiRes and AGASA. Possible reasons range from a systematic error in the energy determination (which would also help explaining the systematic differences in the energy fluxes between the two experiments) to possible calibration problems, due to the adoption of different detection techniques (ground array versus fluorescence). Sadly, it is not clear whether the HiRes experiment will ever be operational again. The near future holds the promise of larger and better experiments to resolve this puzzle. AUGER is still in the stage of construction but the engineering array has already detected many events. This experiment will certainly clear the doubts on the existence of the ultra-GZK events, not only due to the larger statistics but also due to the adoption of a hybrid technique, consisting of a ground array and some fluorescence telescopes. The end of the operation of AUGER will coincide with the start of the EUSO telescope project, the first attempt to measure the flux of ultra-high energy cosmic rays from space. This experiment will be able to pinpoint the sources of these particles. This will go a long way towards settling whether there is a GZK cut off in the cosmic ray spectrum, or not!

\section{References}

Abu-Zayyad et al. (2002), submitted to Phys. Rev. Lett. (astro-ph/0208243)

Abu-Zayyad et al. (2002), submitted to Astropart. Phys. (astro-ph/0208301)

\section{Gravitational Wave Detectors}

Several Laser interferometric gravitational wave detectors, whose objective is to first accomplish direct detection of gravitational waves and then to open a new window of observation to understand astrophysical processes, are in their final stages of completion. They will search for gravitational waves from the collapse of stellar cores resulting in the formation of neutron stars or black holes, and accompanied by a supernova explosion; collisions and coalescence of compact objects such as neutron stars and black holes; the wobbly rotation of neutron stars with deformed crusts; and the relic of gravitational radiation created by a population of astrophysical sources in the early Universe.

Several long-baseline interferometric detectors have now completed the installation phase and have begun taking scientific data. The $300 \mathrm{~m}$ Japanese detector TAMA at Tokyo has been functional for some time. "Data Taking 6" had a total of 1038 hours of 
data and a duty cycle of $86.5 \%$. At present, "Data Taking 7" has a total of 24.6 hours data. The best sensitivity achieved so far is $\left(1.0 \times 10^{-18} \mathrm{~m} / \operatorname{sqrt}(\mathrm{Hz})\right)$.

Laser Interferometer Gravitational-Wave Observatory (LIGO) consists of two four $\mathrm{km}$ long interferometers, one each at Livingston and Hanford, and an additional $2 \mathrm{~km}$ one at Hanford. In January, 2002 LIGO operated all three interferometers simultaneously during a 16-day engineering run dubbed E7. It was the most extensive test conducted of the interferometers thus far and provided an opportunity to test interferometer performance, assess the reliability of the hardware and software during sustained operation, and to test the data handling and data analysis hardware, software, and methods. During the run, the ALLEGRO resonant bar detector at Louisiana State University and the GEO 600 laser interferometer near Hannover, Germany, were also in operation. The LIGO and GEO systems are at the TAMA sensitivity already.

LIGO and GEO began their Science run (dubbed S1) this summer. The two projects ran in coincidence during August 23 to September 9. As compared to the E7 run, the detectors improved their performance by about a factor of 10-100 in a frequency band of $100 \mathrm{~Hz}-4 \mathrm{kHz}$, and acquired the best gravitational wave data ever in the frequency band. GEO achieved an impressive $\mathbf{9 8 . 5 \%}$ duty cycle during this run. The sensitivity is still about two orders of magnitude less than the designed goal. Improvements in detector subsystems over the next year is expected to push the sensitivities toward the desired limit. Data from the coincident runs is currently being analyzed to set useful upper limits on gravitational waves from celestial sources.

VIRGO is just finishing the construction of its $3 \mathrm{~km}$ vacuum arms, and completed the Central Interferometer with its advanced low-frequency seismic attenuation chains and its hierarchical mirror control system. VIRGO plans to commission its long arms as early as the end of this year. Once VIRGO operates as a complete detector it will lead in sensitivity below $50 \mathrm{~Hz}$.

Acknowledgments. The table listing various space missions has been adapted from one originally made by Willem Wamsteker (with a few additions and minor corrections). I wish to express my appreciation to Willem for this important contribution. My sincere thanks are also due to L. I. Gurvits (JIVE, Dwingeloo) for preparing the status report on VSOP, to S. Turck-Chièze, Member of Working Group on Astroparticle Physics) for the news item on the Solar neutrino puzzle, to Pasquale Blasi (Arcetri Observatory, Firenze), for the write-up on Ultra High Energy Cosmic Rays and B. R. Iyer (Raman Research Institute, Bangalore) for the status report on the Gravitational Wave Detectors. 